\title{
APRENDIZAJE ONLINE: SATISFACCIÓN DE LOS UNIVERSITARIOS CON EXPERIENCIA LABORAL
}

\section{ONLINE LEARNING: SATISFACTION OF UNIVERSITY STUDENTS WITH WORK EXPERIENCE}

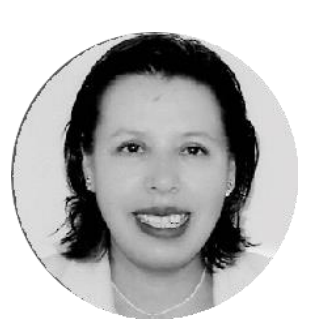

\section{Lida Vásquez-Pajuelo}

Código ORCID: 0000-0002-1241-3190

Profesora deMetodologías dela Investigación

Universidad Nacional del Callao (UNAC) Lima, Perú

Correo electrónico: lidavasquez@gmail.com

Recibido: 22 de junio de 2019

Aprobado: 12 de agosto de 2019

Cómo citar este artículo:

Vásquez-Pajuelo, L. (2019). Aprendizaje online:

satisfacción de los universitarios con experiencia

laboral.

Review of Global Management, 5(2), 28-43.

\section{RESUMEN}

En esta reseña de investigación se presentan los resultados del análisis, la explicación y la aplicación del concepto de satisfacción por el aprendizaje online en un programa de carreras universitarias desarrollado para personas con experiencia laboral. Es un estudio descriptivo univariable, basado en un enfoque mixto, de tipo intersubjetivo, realizado a través de la recolección de datos con encuestas y entrevistas virtuales. Se emplea la plataforma Blackboard Learn, la que también constituye el ambiente virtual para el aprendizaje. Los instrumentos de recojo de información se aplican a través del mencionado medio tecnológico a los 801 estudiantes del año 2014 de cuatro carreras: Negocios Internacionales, Marketing y Gestión Comercial, Administración de Empresas y Contabilidad. 
Los resultados obtenidos indican que se ha validado la variable del estudio, la satisfacción por el aprendizaje online, para el programa "Carreras universitarias para personas con experiencia laboral" (CPEL), basado en la aplicación de una metodología práctica para su medición.

Palabras clave: enseñanza online, estudiantes adultos, experiencia laboral, satisfacción por el aprendizaje.

\section{ABSTRACT}

This research review presents the results of the analysis, an explanation and an application of the concept of satisfaction for online learning in a university degree program developed for people with work experience. It is a univariable descriptive study, based on a mixed, intersubjective approach, type, carried out with data collection through surveys and virtual interviews.

The Blackboard Learn platform is used, which is the actual virtual environment for learning. The information gathering instruments are applied, using the aforementioned technological means, on the 801 students of the year 2014, majoring in International Business, Marketing and Commercial management, Business Administration and Accounting. The results obtained indicate that the study variable, satisfaction with online learning, has been validated for the "University Programs for People with Work Experience" (CPEL, in Spanish), based on the application of a practical methodology for its measurement.

Keywords: online teaching, adult students, work experience, satisfaction with learning. 


\section{INTRODUCCIÓN}

En el ámbito educativo universitario, la universidad recibe progresivamente una mayor demanda de personas que laboran y necesitan desarrollar nuevas habilidades profesionales. Ellas constituyen una población que posee características distintas de los jóvenes ingresantes y provenientes de la escuela y bajo el enfoque del sistema de enseñanza superior tradicional. Esto plantea que las universidades requerirán introducir cambios en las modalidades utilizadas en el proceso de enseñanza-aprendizaje.

En el contexto actual mundial, el empleo de las TICs, debido a su adaptabilidad, ha permitido estrechar la brecha entre el servicio educativo universitario y la demanda mencionada. En este contexto de cambio, se revisan las modalidades virtuales y b-learning (o blended) adoptadas (Domínguez et al., 2015). Así, Gómez y Macedo (2011), resaltan la importancia de los programas virtuales en la educación superior peruana, resaltando su impacto dentro de la dinámica de la educación superior, en relación con el cambio y la adaptabilidad a un mundo globalizado. Desde la perspectiva del estudiante, Martín (2012), propone factores que influyen en la satisfacción de los alumnos que aprenden online, y recoge de ellos que dan mayor importancia a la metodología y planificación docente.

En relación con los componentes que serán modificados para atender dicha demanda, para definir la modalidad virtual, Anuies-Unesco (2004), recalca la mediación del proceso de enseñanza aprendizaje a través de las redes y computadoras, donde los estudiantes y docentes, es decir, los actores del proceso interactúan. Por ejemplo, los estudiantes organizan sus horarios y lugar, con el monitoreo de un tutor (Ho \& Dzeng, 2010).

El otro componente del proceso, los contenidos, se presenta en formatos electrónicos. Estos formatos propician que las actividades o interacciones entre docentes y estudiantes sean colaborativas y en función de formas de comunicación sincrónica y asincrónica (e.g., chat, foros y correo). 
En esta investigación se aplica la definición de Hill (1996), de satisfacción, como las percepciones del cliente o usuario, de que un proveedor o servicio ha alcanzado o superado sus expectativas, de la mano con Cantú (2001), ambos con la misma perspectiva de base, referida a las reacciones personales sobre un producto o servicio.

En este sentido, hay dos vertientes para el estudio de la satisfacción. Una de carácter sicológico, pues es un fenómeno personal; la otra, al considerar la relación con el bien y servicio proporcionado, proviene del campo de la gestión, bajo la denominación de "satisfacción del cliente". La inclusión de las TICs en el aula no es solo tener computadoras: es abrir la puerta a oportunidades de desarrollo y aprendizaje (Díaz, Gutiérrez, Yuste, Arias, Cubo \& Diogo, 2014).

Con respecto al ámbito de investigación, el programa Carreras Universitarias para Personas con Experiencia Laboral (CPEL) de la Universidad San Ignacio de Loyola (USIL), en el Perú, por ejemplo, responde a la necesidad de profesionalización para personas con una experiencia laboral importante. Justamente porque estas personas continúan laborando, el programa ofrece cuatro carreras universitarias completamente virtualizadas, que desarrollan una formación académica de calidad adecuada a sus horarios. El programa cumple las exigencias de la acreditadora americana ACICS 1 .

El programa parte de fundamentos pedagógicos construccionistas y se basa en el fomento de la interactividad, recreando una escenografía en tiempo real, donde la comunicación virtual fluye entre docente-estudiante, estudiante- docente y estudiante-estudiante, en función de la multiculturalidad peruana, lo que promueve actitudes que conllevan la innovación, creatividad, criticidad, reflexión y análisis. Se utilizan medios como redes sociales, $\mathrm{LMS}^{2}$, repositorios y OVA3 ${ }^{3}$, entre otros, que se adapten a las necesidades personales de los participantes profesionales (Castaño, Duart \& Sancho-Vinuesa, 2015; Santacana, 2013). Se emplea la plataforma Blackboard Learn, para hacer posible la convergencia de medios en la gestión de contenidos virtuales de las cuatro carreras del programa: Administración de Empresas, Marketing y Gestión

${ }^{1}$ Accrediting Council for Independent Colleges and Schools

${ }^{2}$ Learning Management Systems.

${ }^{3}$ Objetos virtuales de aprendizaje. 
Comercial, Negocios Internacionales y Contabilidad.

El estudio se propone detectar y describir la satisfacción del aprendizaje online aplicando dos técnicas: la encuesta y la entrevista de satisfacción online. Se explora la satisfacción del aprendizaje online de los estudiantes y se contextualiza el estudio sobre el aprendizaje online brindado en el programa CPEL-USIL de los estudiantes que han cursado alguna asignatura online durante el periodo 2014. Por otro lado, se asume que los cursos online dependen del interés, habilidad, destreza y madurez de los estudiantes, un grupo de estudiantes adultos, que participan de la modalidad online.

La investigación se encuentra motivada con el propósito final de llegar a conclusiones que orienten posteriormente la posible toma de decisiones para mejorar los cursos e-learning y elevar el nivel de satisfacción de los estudiantes.

\section{MÉTODO}

El estudio es descriptivo y univariable, basado en un enfoque mixto de tipo intersubjetivo. La factibilidad del estudio mixto, considerando la población, muestra, recolección y análisis de datos, se plantea dentro de la teoría fundamentada. La población del estudio abarcó la totalidad de los estudiantes participantes en el programa del año 2014, que fue de 801. Para ello, se aplicó un cuestionario en línea a toda la población y se desarrollaron entrevistas

a 12 estudiantes. Mediante métodos de recolección de datos se realiza un análisis de perspectivas, emociones, prioridades, experiencias, significados, es decir, el universo subjetivo en convergencia con la interacción del individuo y la plataforma e-learning. Esto es requerido para captar la doble vertiente del concepto de satisfacción del aprendizaje online.

La variable objeto de la investigación se denomina "satisfacción por el aprendizaje online", y se desagrega en tres categorías: pedagógica, tecnológica y de servicios educativos, que son las que estructuran el constructo teórico. 
Se plantean cinco indicadores y un total de 26 ítems para guiar el análisis del cuestionario. Para la entrevista, se toman en cuenta nueve ítems abiertos que orientarán la aplicación y su posterior interpretación. Se describe el programa CPEL porque condiciona la metodología. Este programa ha sido diseñado para personas adultas. A la fecha del estudio, los estudiantes se encuentran en el rango de edad de entre 20 y 61 años, con cuatro años de experiencia laboral mínima. Este diseño se traduce en la temporalidad del proceso de enseñanza-aprendizaje, la estructura modular, el sistema de evaluación y la metodología aplicada en el proceso de aprendizaje online.

En el aspecto metodológico, las sesiones de aprendizaje se desarrollan mediante aprendizaje basado en casos, en los que se motiva al estudiante a verter sus conocimientos previos (empíricos). Con la orientación del docente, contrastan dicho conocimiento con las bases teóricas para una aplicación inmediata y eficiente; y se logra de esta manera un aprendizaje significativo y consistente.

En la plataforma Blackboard Learn se publican los materiales de contenidos temáticos (e.g., materiales de consulta obligatoria, material complementario y recursos de apoyo) y se usan herramientas interactivas, como foros temáticos o de discusión, documentos compartidos, anuncios, actividades individual grupales y videoconferencias a través de Blackboard Collaborate. El diseño de las actividades de aprendizaje se basa en las potencialidades de las herramientas info-comunicacionales: la plataforma Blackboard Learn para la gestión del contenido, y la plataforma Moodle, para realizar evaluaciones online, lo que permite un encuentro virtual docenteestudiantes y estudiantes- estudiantes del curso para el trabajo cooperativo, la discusión y el intercambio.

\section{RESULTADOS}

La variable de satisfacción por el aprendizaje online se mide en relación 
con tres categorías: el enfoque pedagógico, el enfoque tecnológico y el enfoque de servicios educativos. Ellas todas vinculadas respectivamente al proceso de enseñanza-aprendizaje; a los medios y materiales del proceso; y a la gestión institucional del proceso, más una categoría de control (i.e., preguntas de complemento).

Cada categoría se desagrega en indicadores. Así, la categoría de enfoque pedagógico, que es la más importante por su contenido, se divide en dos indicadores: los cursos virtuales del programa CPEL y el servicio docente. Estos indicadores están referidos, respectivamente, a los contenidos del proceso de enseñanza-aprendizaje y a las actividades e interacciones en dicho proceso. Las otras tres categorías sólo corresponden a un indicador por cada una: soporte y plataforma Blackboard Learn, los servicios administrativos y las preguntas de complemento, que corresponden respectivamente a los medios y materiales, a la gestión institucional y al control de la fiabilidad del instrumento.

Cada indicador se desagrega en ítems, y, además, hay un ítem referido al indicador de manera global. El contraste entre lo específico y lo general del indicador permite evidenciar diferencias de percepción y controlar la fiabilidad de las respuestas. Por otro lado, se establece, en cada pregunta, una escala de cinco niveles: muy insatisfecho; insatisfecho; poco satisfecho; satisfecho; y muy satisfecho. Entre estos cinco niveles, sólo los dos últimos indican satisfacción. Por lo tanto, en términos de porcentaje, sólo se valida la variable de manera positiva si los estudiantes responden en uno de los dos últimos niveles.

En el caso del primer indicador de la categoría de enfoque pedagógico, cursos virtuales del programa CPEL, el promedio de los ítems específicos resulta de $77.84 \%$ de validación de la variable, mientras que la respuesta global del indicador es de 78.78\%, ver Tabla 1. Esto da una diferencia no significativa entre ambos (0.94). Por ello, se podría afirmar que se da por válida la satisfacción por el aprendizaje online respecto de los contenidos del programa. 
Tabla 1:

Satisfacción por el aprendizaje de los cursos online.

Categoría de enfoque pedagógico

\section{Aspectos analizados}

Satisfacción

Organización de la estructura de los cursos en general $78,2 \%$

Calidad del contenido de las diapositivas, lecturas, videos y casos $79,1 \%$

Lenguaje utilizando en los materiales de los cursos $76 \%$

Actividad deaprendizaje pararelacionarlocon el tema de estudio $80 \%$

Cantidad de actividades de aprendizaje programada $74,6 \%$

Evaluación y su relación con los temas desarrollados $78,9 \%$

Nivel de satisfacción general de los cursos

$78,7 \%$

Fuente: Satisfacción del aprendizaje online. Parte de una tesis doctoral realizada por Vásquez, 2015.

En el caso del segundo indicador de la categoría, el promedio de los ítems específicos resulta de $70.86 \%$ de validación de la variable, mientras que la respuesta global para el indicador es de $74.78 \%$, lo cual da una diferencia no significativa entre ambos (3.92). Por ello, sería posible afirmar que se da por válida la satisfacción por el aprendizaje online respecto de las actividades del programa, ver Tabla 2.

\section{Tabla 2:}

Satisfacción por el aprendizaje de los cursos online.

Categoría de enfoque pedagógico

\section{Aspectos analizados}

Respecto del tiempo de respuestaa consultas en los foros académicos

Respecto de las respuestas obtenidas

Con el seguimiento de las actividades de aprendizaje

Sobre la retroalimentación de las evaluaciones

Con la comunicación del docente (tutor-virtual)

Nivel de satisfacción general del desempeño docente virtual

\section{Satisfacción}

$71,91 \%$

$72,03 \%$

$71,04 \%$

$66,42 \%$

$72,91 \%$

$\mathbf{7 7 4 , 7 8 \%}$

Fuente: Satisfacción del aprendizaje online. Parte de una tesis doctoral realizada por Vásquez, 2015. 
En resumen, se puede evidenciar satisfacción por el aprendizaje online respecto del proceso de enseñanza-aprendizaje.

En cuanto a la segunda categoría, el enfoque tecnológico, dado que solo hay un indicador, el promedio de los ítems específicos resulta de 58.85\% de validación de la variable, mientras que la respuesta global para la categoría es de 70.91\%, lo cual da una diferencia de 12.06 entre ambos, que es significativa- ver Tabla 3. Por ello, no sería posible validar de manera inequívoca la satisfacción por el aprendizaje online en cuanto a la relación con la plataforma del programa, es decir, a los medios proporcionados por el programa, dado que hay una discrepancia entre la percepción global y la percepción de los aspectos específicos.

Tabla 3:

Satisfacción por el aprendizaje de los cursos online.

Categoría de enfoque pedagógico

\section{Aspectos analizados}

Satisfacción

Capacitaciónadecuadaparaeluso delaplataformadeaprendizaje virtual (Blackboard)

Con el acceso y navegación en el curso $56,43 \%$

Conlainteracciónque ofrecelaplataformaparacomunicarsecon compañerosydocente

Intervencióndelequipodesoportevirtualadudasoproblemas en eluso dela plataforma

Fiabilidad de la plataforma Blackboard

Nivel de satisfacción general del desempeño docente virtual

Fuente: Satisfacción del aprendizaje online. Parte de una tesis doctoral realizada por Vásquez, 2015.

Con relación a la tercera categoría, enfoque servicios educativos, dado que solo hay un indicador, el promedio de los ítems específicos resulta de $73.04 \%$ de validación de la variable, mientras que la respuesta global para la categoría es de $74.78 \%$, lo cual da una diferencia de 1.74 entre ambos, que no es significativa- ver Tabla 4. Por ello, se podría afirmar que se da por válida la satisfacción 
por el aprendizaje online respecto de la gestión institucional del programa.

\section{Tabla 4:}

Satisfacción por el aprendizaje de los cursos online.

Categoría de enfoque pedagógico

\section{Aspectos analizados}

Satisfacción

Apoyo brindado al estudiante por el Centro de Atención Virtual $70,54 \%$ (CAV)

Apoyo brindado al estudiante en la gestión de $74,16 \%$ trámites administrativos: matrícula, retiros, traslados, pagos, etc.

Comunicación oportuna de los procesos administrativos

Nivel de satisfacción general del desempeño docente virtual

Fuente: Satisfacción del aprendizaje online. Parte de una tesis doctoral realizada por Vásquez, 2015.

Se agregó, como otra forma de confirmación de la fiabilidad de las respuestas, un indicador e ítems complementarios que plantean consecuencias conductuales y valoraciones en referencia a la satisfacción, así como una pregunta global también para el indicador. La diferencia entre el promedio de las preguntas específicas y la global es no significativa (1.75), por lo que se consideraría fiable la afirmación sobre la validez del grado de satisfacción señalado-ver Tabla 5.

\section{Tabla 5:}

Satisfacción por el aprendizaje de los cursos online.

Categoría de enfoque pedagógico

\section{Aspectos analizados}

Satisfacción

¿Recomendaría el curso virtual a sus compañeros,

$72,28 \%$

amigos o colegas?

¿Considera que el curso cumple con su formación

$75,78 \%$

integral como profesional?

Nivel de satisfacción general del desempeño docente virtual

Fuente: Satisfacción del aprendizaje online. Parte de una tesis doctoral realizada por Vásquez, 2015. 
Con respecto a los resultados de la entrevista a 12 estudiantes acerca de la variable, en general se confirmaría el nivel de satisfacción evidenciado en lo anterior, con una diferencia en la categoría de enfoque tecnológico, que fue valorada positivamente de manera unánime en función de sus posibilidades, sobre todo, antes que en las conductas realizadas.

\section{DISCUSIÓN}

La investigación realizada bajo un enfoque mixto, descriptivo, de tipo inter- subjetivo, se basó en el análisis de la descripción de la actividad de los partici- pantes de los cursos. Se realizó bajo la modalidad online del programa CPEL, cuyos estudiantes poseen la capacidad económica para trabajar en una plataforma virtual, en virtud del equipamiento al que acceden. Con ello, se busca neutralizar el efecto de aspectos limitantes, en la medida en que todos los estudiantes dispondrán de las mismas posibilidades de acceso a la plataforma Blackboard Learn usada como medio.

En definitiva, la satisfacción del aprendizaje online estaría validada por las personas consideradas en el estudio. Esto supone una valoración subjetiva del éxito alcanzado por la institución; ello por cuanto no parte de criterios concretos y objetivos, sino más bien del enfoque hacia las percepciones y actitudes frente a las condiciones que se les brinda a los estudiantes (Gordillo, Rodríguez \& Sanchiz, 2014).

Al ser la satisfacción dependiente del punto de vista del estudiante, y al manifestarse como percepción de bienestar ante los servicios ofrecidos, en interacción con los intereses del estudiante, resulta un concepto elusivo por su subjetividad, pero que es de relevancia para la evaluación de los programas y servicios educativos, en especial los virtuales. Además, se encuadra dentro del concepto de satisfacción del cliente, que implica una perspectiva de oferta-demanda que debe ser considerada. 
La satisfacción es un componente psicológico que motiva la continuidad en el estudio y en el servicio, por lo que incide en la calidad medible de la universidad. La metodología empleada para el análisis de los datos proporcionados por los estudiantes del programa CPEL permitió no sólo evidenciar el grado de satisfacción, sino también contrasta la fiabilidad respecto de declaraciones subjetivas. De este modo, es posible ubicar el origen de dicho nivel de satisfacción.

Además, esto puede localizarse conceptualmente desde la perspectiva del construccionismo, que destaca la importancia de la acción, que parte del supuesto de que, para que se produzca el aprendizaje, el conocimiento debería ser construido (o reconstruido), por el propio sujeto que aprende a través de la acción y la interacción mediante las redes con sus pares, con el docente y con toda la comunidad disponible. En este caso, la satisfacción habría sido relacionada con los diversos componentes del proceso de enseñanza-aprendizaje, con lo que se ofrece un mapeo que posibilitaría determinar el ámbito donde aparecen inconvenientes o dificultades que generen un bajo nivel de satisfacción.

La contundencia de los resultados, que llega a un $78.7 \%$ de estudiantes satisfechos con la forma de desarrollarse estos cursos, se explicaría a partir de lo hallado por Gómez y Macedo (2011), quienes en su estudio dan a conocer la importancia de los programas virtuales dentro de la dinámica de la educación superior, especialmente en lo metodológico y didáctico, pues consideran que esto ha permitido la incorporación de herramientas web, que cautivan en cierta forma a los estudiantes con sus nuevas formas de aprender. Una de ellas es internet y las herramientas que allí se encuentran (Siemens \& Fonseca, 2004).

Asimismo, pudo conocerse que el grado de satisfacción con respecto al soporte y plataforma Blackboard Learn en la población de personas adultas alcanzó al 70.9\% de los estudiantes y al 74.7\% de estudiantes satisfechos con el servicio docente. En tal sentido, esto coincide con Salgado (2015), quien estudió la enseñanza y el aprendizaje en modalidad virtual y encontró que los estudiantes demostraron en general un nivel adecuado de satisfacción 
con los cursos virtuales, en un grado equivalente a los cursos presenciales de la facultad y de la universidad como un todo. Principalmente por el orden o estructura con que el docente implementa el curso; la retroalimentación que ofrece el docente; la "cordialidad" del docente, o grado en que motiva, orienta y se identifica con el grupo; así como un nivel óptimo de exigencia académica.

Sin embargo, se mediatizaría esta conclusión en relación con una discrepancia entre las respuestas específicas y las globales. En este sentido, es rescatable de la entrevista el contraste entre una valoración basada en las conductas efectivas, medida por el cuestionario, y una valoración a partir de las posibilidades de la plataforma, que se manifiesta como efecto de la influencia social. Esto último puede ser objeto de próximas investigaciones, tomando en cuenta modelos de aceptación de tecnología, como la teoría unificada de la aceptación y uso de la tecnología (UTAUT), propuesta por Venkatesh et al. (2003), que hoy se encuentra en su tercera versión (Venkatesh et al., 2016), aplicada también a la medición de la satisfacción en relación con adopción de tecnologías.

\section{CONCLUSIÓN}

El análisis de la importancia de la enseñanza online para estudiantes adultos y con experiencia laboral ha permitido arribar a los siguientes resultados, bajo un enfoque mixto, descriptivo, de tipo intersubjetivo, en que se usaron dos técnicas. La primera fue la técnica directa con el instrumento de entrevista estructurada de nivel profundidad para el recojo de información y su respectivo análisis categórico de los resultados. La segunda técnica, fue la técnica indirecta, la encuesta, con su instrumento, el cuestionario. Se llegó al punto de la saturación, triangulando la información y a una serie de los resultados, en su mayoría esperados.

Es evidente que los estudiantes sienten que desarrollan competencias, que movilizan sus aprendizajes; y que valoran el nivel de desempeño alcanzado con la satisfacción por el aprendizaje online aprobando sus cursos. 
A ello se suma el grado de satisfacción con respecto a los servicios administrativos, con el $74.7 \%$ de los estudiantes, indicador de la buena y pronta atención de sus necesidades.

La satisfacción por el aprendizaje online es un aporte de la investigación, que evidencia que la formación académica virtual garantiza y se constituye en un referente para la versión virtual de las universidades; y contribuye en la formación de personas adultas que trabajan para concluir su sueño de ser profesionales (Salgado, 2015).

El análisis de los resultados valida la satisfacción respecto del programa CPEL en $78.7 \%$ de estudiantes satisfechos, lo que demuestra la opinión de más de las tres cuartas partes de los estudiantes encuestados.

El nivel de satisfacción por el enfoque tecnológico que se da en el programa tiene doble vertiente, según se asuma como base las conductas efectivas o la valoración por prospectiva.

Con respecto a los servicios educativos, los resultados muestran que la proporción de estudiantes satisfechos es similar a lo obtenido en las otras categorías. En ellos se reporta un $74.78 \%$ de estudiantes satisfechos con los servicios administrativos.

\section{REFERENCIAS}

Anuies-Unesco.(2004). La educación superior virtual en América Latina y el Caribe. México: Anuies.

Castaño,J., Duart,J.M., \& Sancho-Vinuesa, T. (2015). Determinantes del uso de interactivo: un estudio exploratorio. New Approaches in Educational Research, 4(1),2534. 
Díaz, L., Gutiérrez, P., Yuste, R., Arias, J., Cubo, S., \& Diogo, A. (2014).Usos deaulas virtuales síncronas en educación superior.Pixel-Bit. Revista de Medios y Educación, 45,203-215

Domínguez, H., Antonio, J., Bárcenas, L., Estrada, M., Tolosa, S., \& José, S. (2015). Hacia las competencias digitales en la actividad docente: un diagnóstico para definir los elementos fundamentales. Ciudad de México: Universidad Nacional Autónoma de México.

Gómez, L، \& Macedo,J.(2011). Importancia delos programas virtuales en la educación superior peruana. Investigación Educativa, 15(27), 113-126.

Gordillo,J.J. T,, Rodríguez, V.H. P., \& Sanchiz, D. C. (2014). Investigación evaluativa de la formación online en prevención de riesgos laborales y medio ambiente: unanálisis cualitativo delos programasimpartidos por la Fundación Istas. Revista de Investigación Educativa, 32(1),73-90.

Gutiérrez-Santiuste, E. (2012). Comunicación en entornos virtuales de formación: estudio de la interacción didáctica en diversas modalidades de enseñanza-aprendizaje en educación superior (tesis doctoral). Universidad de Granada: España.

Hernández, R., Fernández, C., \& Baptista, P. (2014). Enfoque cualitativo. Metodología de la investigación (6.a ed.). Ciudad de México: McGraw-Hill.

Ho, C. L. \& Dzeng, R. J.(2010). Construction Safety Training Via e-Learning: Learning Effectiveness and User Satisfaction. Computers \& Education, 55(2), 858-867.

Martín,0.(2012).La satisfacción del usuario en la enseñanza virtual: el caso delsistema universitario andaluz(tesisdoctoral).Universidadde Granada: España. 
Salgado, E. (2012). Enseñanza para la comprensión en la educación superior: la experiencia de una universidad costarricense. Revista Iberoamericana de Educación Superior, 3(8), 34-50.

Salgado, E. (2015). Diálogo y aprendizaje percibido en estudiantes de modalidad virtual:abordaje cualitativo en un programauniversitario en Costa Rica. Revista Iberoamericana de Educación a Distancia, 18(1),191-211.

Salgado, E.(2015). La enseñanza y el aprendizaje en modalidad virtual desde la experiencia de estudiantes y profesores de posgrado de la Universidad Latinoamericana de Ciencia y Tecnología (tesis doctoral). Universidad Latinoamericana de Ciencia y Tecnología: Costa Rica.

Siemens, G. \& Fonseca, D. E. L. (2004). Conectivismo: una teoría de aprendizaje para la era digital. Recuperado de http://www.fce.ues.edu.sv/uploads/ pdf/siemens-2004-conectivismo.pdf

Ruiz, A. B. M., García-Sánchez, F. A., \& Sánchez-López, M. C. (2011). Implicación del alumnado en la valoración de su satisfacción con las webs didácticas. Edutec. Revista Electrónica de Tecnología Educativa, (37).

Venkatesh, V., Morris, M. G, Davis, G. B., \& Davis, F. D. (2003). User Acceptance of Information Technology: Toward a Unified View. MIS quarterly, 425478. Recuperado de http://www.jstor.org/stable/30036540

Venkatesh, $\mathrm{V}_{\text {, }}$ Thong,J.Y, \& Xu, X. (2016). Unified Theory of Acceptance and Use of Technology: A Synthesis and the Road Ahead.Journal of the Association for Information Systems, 17(5),328. Recuperadodehttp:// search.proquest.com/openview/e094d55bdfb4fb7441195bf9d0363f5 d/1?pq-origsite $=$ gscholar \&cbl $=26427$

Wang Y. S. (2003). Assessment of Learner Satisfaction with Asynchronous Electronic Learning Systems, 41(1), 75-86. 